\title{
UM FRAMEWORK DE COMUNIDADES DE PRÁTICA EM AMBIENTES VIRTUAIS DE APRENDIZAGEM
}

\author{
Mauro Fiorio $^{1}$, João L. T. da Silva ${ }^{1}$, Alexandre M. Ribeiro ${ }^{1}$ \\ ${ }^{1}$ Centro de Computação e Tecnologia da Informação - Universidade de Caxias do Sul \\ (UCS) \\ Caxias do Sul - RS - Brazil \\ fioriomegmail.com, jltsilva@ucs.br, aribeiro@ucs.br
}

\begin{abstract}
Resumo. Este artigo descreve um framework conceitual para especificação de comunidades de prática $(C d P)$ e mostra como ele pode ser utilizado em ambientes virtuais de aprendizagem (AVA). O framework proposto levou em consideração as características dos ambientes virtuais de aprendizagem no contexto de comunidades de aprendizagem. A perspectiva de modelagem de AVAs segundo uma interação virtual em termos de CdPs promove uma ampla interação entre alunos e professores. O framework define a gestão automática da busca e recomendação de comunidades e interesses veiculados por seus participantes.
\end{abstract}

Palavras-chave. Comunidades de Prática, Ambiente Virtual de Aprendizagem, Gestão do Conhecimento.

\section{A FRAMEWORK OF COMMUNITIES OF PRACTICE IN VIRTUAL LEARNING ENVIRONMENTS}

\begin{abstract}
The article describes a conceptual framework for the specification of communities of practice (CoP) and shows how it can be applied into virtual learning environments (VLE). The proposed framework took into account the features of VLE in the context of learning communities. The modeling perspective of VLEs through virtual interactions in terms of CoPs promotes a broad interaction of students and teachers. The framework defines the automatic management of search and recommendation of communities and interests supported by its participants.
\end{abstract}

Keywords. Communities of Practice, Virtual Learning Environment, Knowledge Management.

\section{INTRODUÇÃO}

Frequentemente, trabalho e aprendizagem estão vinculados, de forma virtual, a Ambientes Virtuais de Ensino-Aprendizagem (AVA). Ambientes de ensinoaprendizagem tradicionais geralmente são vinculados a Sistemas de Gestão da Aprendizagem (LMS) e funcionam a partir de portais corporativos e/ou acadêmicos, fazendo uso extensivo de ferramentas de colaboração. As ferramentas de comunicação encontram-se bem desenvolvidas e já atendem aos requisitos básicos de uma comunidade. Entretanto, ao centralizar a atenção em determinados domínios ou indivíduos, o poder de compartilhamento de conhecimento é reduzido, dificultando a formação dinâmica de comunidades de aprendizagem. Logo, as ferramentas tradicionais de comunicação e colaboração precisam de uma reformulação de uso, passando de um ambiente centralizado e estático, para a troca aberta e dinâmica de conhecimento em grupos auto-organizáveis.

Nesta nova perspectiva, o uso das comunidades assume um papel relevante em termos de aprendizado e manutenção do conhecimento comum a um grupo de pessoas e mesmo a uma organização. $\mathrm{O}$ fato de comunidades se formarem de maneira dinâmica e espontânea determina a evolução das possibilidades das ferramentas de colaboração em portais colaborativos e redes sociais. Estas últimas assumem um caráter ainda mais comunitário se levarmos em conta a quantidade de cruzamentos e análises de informações veiculadas pelos seus servidores e provedores de serviços. 
Portanto, um dos grandes interesses gerais na aprendizagem organizacional hoje em dia, encontra-se em conjuntos de pessoas que se reúnem por um interesse comum em aprender e aplicar práticas de forma espontânea, compartilhando e absorvendo conhecimento e experiência com outras pessoas. Esta ideia define o que Wenger (2008) chamou de Comunidades de Prática (CdP), que também podem ser informalmente caracterizadas como grupos de pessoas que compartilham um conceito ou uma paixão por algo que elas fazem e que interagem regularmente para aprender a fazê-lo melhor (Terra, 2005).

Entretanto, o uso de CdPs aponta ainda o pouco suporte que as Tecnologias de Informação e Comunicação (TIC) podem oferecer ao gerenciamento de comunidades mantendo a característica de $\mathrm{CdP}$ e promovendo a troca de conhecimento. Requisitos como criatividade, autonomia, criticidade e colaboração, embora oferecidas pela maioria das ferramentas de TICs não oferecem grandes funcionalidades de organização que promovam o conhecimento coletivo.

Este artigo apresenta uma estrutura para dar suporte à criação de CdPs em um Ambiente Virtual de Aprendizagem, resultando em um "framework conceitual" que estabelece as principais funcionalidades e interações necessárias entre as ferramentas tradicionais de AVAs e CdPs, bem como as interações entre usuários de ambos. $\mathrm{O}$ artigo está organizado em seis seções. As seções 2 e 3 apresentam uma breve revisão sobre comunidades de prática e ambientes virtuais de aprendizagem no contexto do presente estudo. A seção 4 apresenta uma visão resumida do Framework conceitual para Comunidades de Prática. A seção 5 descreve um protótipo e um experimento realizado com uma análise inicial do uso no contexto de aprendizagem. A seção 6 finaliza o artigo apresentado algumas lições aprendidas e contribuições deste trabalho.

\section{COMUNIDADES DE PRÁTICA}

Segundo Etiene Wenger (2008) as comunidades de prática apresentam três características fundamentais: domínio, comunidade e prática. O Domínio representa o tema ou assunto sobre o qual os membros da comunidade compartilham conhecimento e práticas e aprendem mutuamente, reconhecendo o valor coletivo de suas experiências. $\mathrm{O}$ Domínio deve ser explícito e presente tanto na CdP como para os indivíduos, o que traça um ligação forte entre os indivíduos e as comunidades. A Comunidade representa o relacionamento formal entre um grupo de pessoas que participam de discussões e atividades coletivas, ajudando-se mutuamente e compartilhando conhecimento e experiências do domínio proposto. A Prática é o conjunto de recursos e técnicas compartilhadas para a resolução de problemas.

Atualmente, as organizações estão reconhecendo a contribuição das $\mathrm{CdP}$ na geração e compartilhamento do conhecimento, conectando pessoas por meio de redes sociais, identificando competências e sendo modelo de aprendizagem (Kanter, 2001; XEROX, 2005; WORDBANK, 2008; IBM, 2008), (Gongla \& Rizzuto, 2001). No contexto de instituições de ensino, comunidades de docentes aglomeram conhecimento que pode ser agrupado em áreas de competência e interesses (coletivos ou individuais), e ainda orientar uma organização temática regida pela estrutura administrativa e curricular dos cursos. Este cenário constitui então uma grande oportunidade e laboratório para o uso de CdP.

Para Albagli (2007), a utilização das TICs facilita a circulação das informações e 
conhecimentos, porém a capacidade cognitiva e a capacidade de inovação não são afetadas pela tecnologia. Desta forma, as CdP usam ferramentas tecnológicas tradicionais, utilizadas em sites pessoais, corporativos ou de relacionamento, mudando apenas seus objetivos e formas de utilização. Albagli e Wenger estudaram e sugerem várias ferramentas computacionais para instrumentalização de CdPs, desde ferramentas de comunicação tradicionais como chats, fóruns de discussão, faqs, e-mail, mensagens instantâneas, ou ferramentas de comunicação mais aprimoradas como quadros brancos, podcasting, vídeo-conferência (ver Figura 1). Também são necessárias ferramentas de gestão e publicação. Neste contexto, a inovação não está nas ferramentas, mas no enfoque de seu uso, que possibilita aos membros da $\mathrm{CdP}$ a disseminação do conhecimento.

As interfaces requeridas por CdP são identificadas por Wenger et al. (2005) através de tensões presentes nas relações de troca entre os membros e a comunidade gerando três tipos de necessidades que definem possibilidades tecnológicas que visam atender às necessidades dos membros de uma comunidade: Interação (síncronas $e$ assíncronas), Publicação e Tendência (participação individual e cultivo da comunidade), ilustrado no quadro sinóptico bidimensional da Figura 1.

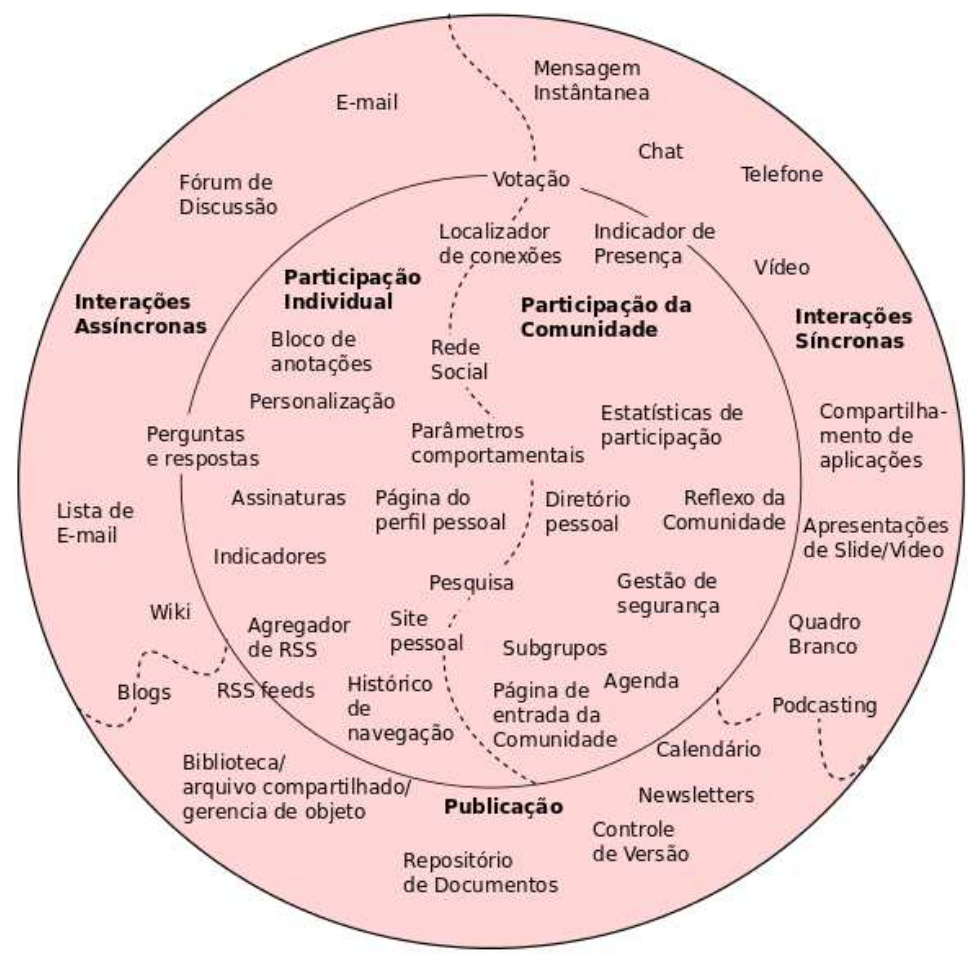

Figura 1: Atividades relacionando ferramentas tecnológicas às tensões encontradas em CdP (Wenger et al. 2005).

A interconexão entre as ferramentas e as CdP são parte central deste trabalho. As comunidades possuem um domínio, que transparece nos diálogos e na própria gerência da comunidade, então se aplicadas metodologias de busca contextual nos registros dos fóruns de discussão, chats, faqs, e outras ferramentas de comunicação, é possível a inferência de interesses dos indivíduos para associá-los às comunidades que apresentam os mesmos interesses.

As relações existentes entre as ferramentas, experiências e interesses pessoais são muitas, o que torna difícil de esgotá-las, mas o objetivo é favorecer a disseminação do conhecimento, ampliar a abrangência das práticas e aumentar as relações, sugerindo 
novas e fortalecendo as existentes.

\section{AMBIENTES VIRTUAIS DE APRENDIZAGEM}

Os Ambientes Virtuais de Aprendizagem (AVAs) constituem-se em um conjunto de ferramentas de TIC que oferecem suporte à aprendizagem. Este suporte pode seguir um estilo de ensino tradicional, presencial, ou emular um modo totalmente à distância através de ferramentas de comunicação e interação via internet. Segundo Casa e Ribeiro (2005), nestes ambientes os papéis tradicionais dos professores e alunos são redefinidos, transformando o aluno em um agente mais ativo no processo de aprendizagem, onde ele produz conhecimento, estabelece relações e colabora com os colegas, socializando ideias.

Segundo Dillenbourg (2000) a interação intensiva entre um grupo de pessoas através de algum meio constitui uma comunidade. Embora o sentimento de comunidade não emerja per se, devido somente à comunicação eletrônica, o sentimento se constitui através de uma grande quantidade de interações e tempo que requerem objetivos e experiências compartilhadas. Para Dillenbourg então, o sucesso de um AVA ou uma comunidade virtual depende de como o meio tecnológico promove o aprendizado e o desenvolvimento do processo cultural entre diferentes indivíduos.

Os ambientes virtuais de aprendizagem podem ser utilizados, sob pelos menos, três formas distintas, uma como ferramenta para o ensino à distância, como suporte ou apoio ao modelo presencial de educação e outra no formato semi-presencial (ou blended). Como ferramenta de ensino à distância, os AVA desempenham o papel de canal de comunicação e ligação entre os alunos e professores, garantindo agilidade nas resoluções de dúvidas, permitindo acesso rápido e atualizado aos conteúdos do domínio estudado.

Foram estudados três ambientes que apresentam suporte à AVAs, tendo como referência suas ferramentas, para a definição das relações com os aspectos do conteúdo e as possibilidades apresentadas por cada ferramenta, conforme seus objetivos. MOODLE, TELEDUC e UCSvirtual apresentam características semelhantes e um conjunto idêntico de ferramentas básicas, as divergências estão relacionadas aos objetivos específicos e as experiências do amadurecimento de cada ambiente.

Os três produtos apresentam pontos fortes nas ferramentas de comunicação, síncronas ou assíncronas, oferecendo diversas opções que garantem interações mais pessoais, dirigidas a indivíduos específicos ou comunicação coletiva para resolução de problemas ou assuntos gerais. Normalmente são ferramentas tradicionais de uso comum a qualquer usuário da web, o que facilita o uso e não requer treinamentos específicos.

As ferramentas voltadas para o gerenciamento dos conteúdos publicados também apresentam grande semelhança e estão presentes em todos ambientes, divergindo apenas na forma de uso.

Os ambientes MOODLE e TELEDUC são genéricos. Não apresentando um enfoque específico a uma instituição, eles devem adaptar-se às características da instituição. Esta vocação generalista necessita da presença de um conjunto amplo de ferramentas, algumas administrativas, para as turmas e outras ferramentas complementares para as atividades.

O AVA do UCSvirtual é proprietário à instituição e apresenta serviços mais voltados ao funcionamento da instituição, alguns são funcionais apenas no contexto 
desta universidade, como a Avaliação On-line e a Matrícula. Ambos apresentam parte do funcionamento no ambiente virtual e parte distribuído pelos softwares de gestão da instituição. No AVA do UCSvirtual as ferramentas relacionadas à atividades dos alunos e turmas não apresentam muitas opções, isto porque o ambiente é uma forma de apoio para as aulas presenciais que fazem todos os controles das atividades a serem desenvolvidas.

De modo geral os ambientes estudados estão voltados para a aprendizagem de algum domínio, investindo em ferramentas para a comunicação, que estão bem desenvolvidas e apresentam grande facilidade de uso e auxílio na aprendizagem pela troca de experiências e resolução de problemas. Entretanto, a centralização das atividades na turma reduz o poder de publicação dos trabalhos dos alunos e mantém os grupos formados restritos a um universo menor do que a instituição. Mesmo existindo ferramentas para formação de grupos nos AVA elas tem enfoque de grupo para realização das atividades da turma, e não de grupos de discussão ou grupos de estudo.

Esta configuração dos AVAs não permite que sejam criadas, por exemplo, grupos virtuais para o amadurecimento de algum domínio. As turmas se formam, realizam suas atividades e dispersam-se. A próxima turma não encontrará referências sobre os estudos da turma anterior, enquanto, se fossem formadas CdP estas poderiam permanecer e tornarem-se referência para este domínio, auxiliando as novas turmas e gerando mais conhecimento que estará carregado de novas experiências e novas práticas.

\section{FRAMEWORK PARA COMUNIDADES DE PRÁTICA}

As CdPs têm um ciclo de vida bem definido, em relação a seus estágios, mas não existem limites no âmbito temporal para definição de cada um dos estágios, definidos por Wenger (1998) como criação, expansão, maturação, atividade e dispersão. O framework conceitual proposto, apresenta os componentes principais que devem ser considerados na utilização de CdP no contexto de um AVA. O diagrama da Figura 2 apresenta o mapa conceitual dos elementos envolvidos no framework, simbolizando os conceitos e suas inter-relações envolvendo uma CdP.

O AVA também possui um conjunto de usuários, alunos, docentes e comunidade externa, que garantem o funcionamento e a dinâmica desta ferramenta, já que sem os participantes, o conhecimento não seria compartilhado. O papel dos participantes é fundamental como concentradores de conhecimento. Estes Indivíduos povoam o AVA e são potenciais candidatos a formarem CdP, conforme seus Domínios de Interesse.

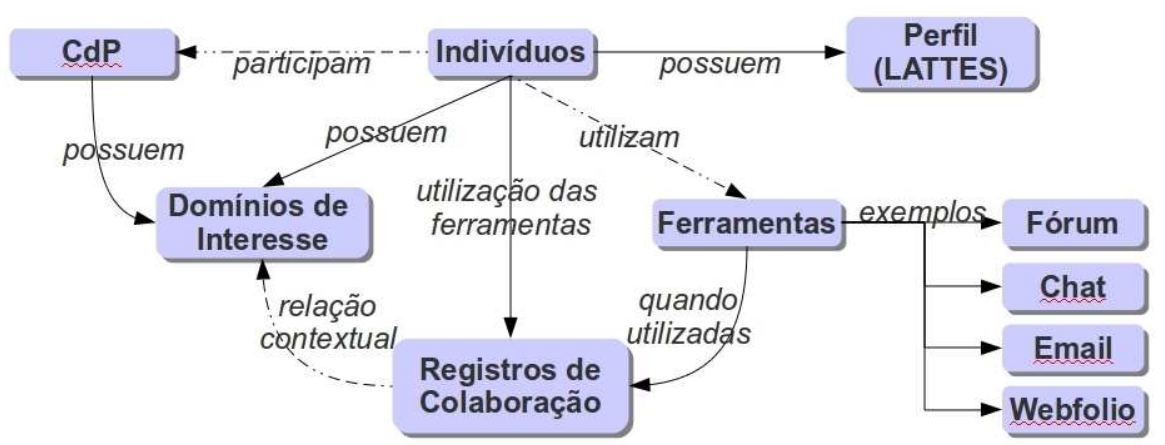

Figura 2: Estrutura diagramática da proposta de um framework para CdP. 
Os Domínios de Interesse, por sua vez, descrevem os participantes quanto às suas aspirações, interesses e necessidades. Entretanto, isso não representa todo perfil do indivíduo, que engloba também os textos e material que são submetidos ao portal, a sua interação nos fóruns de discussão, chats e participações colaborativas além da descrição estática do seu perfil que apresenta uma série de informações complementares, como a formação, as experiências profissionais, títulos e prêmios, áreas e projetos de pesquisa, constituindo assim um Perfil detalhado de cada participante.

A classe CdP contém o conhecimento que emerge de relações informais entre indivíduos, que são detectadas com o auxílio dos Registros de Colaboração, que constituem os registros históricos do uso das ferramentas colaborativas.

\section{ESTUDO DE CASO DO FRAMEWORK NO UCSVIRTUAL}

O estudo de caso ilustra uma arquitetura simples para ser acoplada ao UCSVirtual, a qual não terá necessidade de uma alteração completa do AVA. A arquitetura é composta por três elementos: o AVA, o framework e o banco de dados. O AVA utilizado, UCSVirtual, mantem-se inalterado. O banco de dados foi ampliado para conter os dados do AVA e os registros das interações relacionadas aos assuntos. O framework orienta a criação dos scripts na gestão do banco de dados e estabelece as relações entre os domínios de interesse.

A criação de CdPs é realizada de forma paralela às turmas do AVA, observando os objetos e relacionando-os através de palavras-chave. A troca de conhecimento existente no AVA pode ser utilizada como apoio na identificação dos domínios de interesses. Igualmente os participantes da $\mathrm{CdP}$ possuem perfis que descrevem seus interesses e conhecimentos. Neste experimento, os domínios de interesse estão relacionados a um conjunto de palavras-chave que define um assunto específico e são gerenciados no próprio framework.

No ambiente do AVA diversas interações ocorrem todos os dias, usuários refinam seus perfis, postam perguntas e respostas em fóruns, enviam arquivos que tratam de assuntos específicos, consultam e contribuem com os materiais específicos das disciplinas que estão matriculados. Todas as interações ficam devidamente registradas e podem ser rastreadas através dos registros de colaboração.

A Figura 3 ilustra um mapa exemplificando uma busca por "novos participantes". Os indivíduos A e B possuem diversos domínios de interesse associados pois tem um perfil definido e diversas interações com as ferramentas do AVA, o que também cria associações com "domínios de interesses".

Em algum momento a CdP busca possíveis novos participantes para compartilhar conhecimento e as práticas da própria CdP, esta busca é uma funcionalidade disponível no framework que através dos "domínios de interesses" comuns indica indivíduos que compartilham da "paixão" pelo domínio da CdP. 


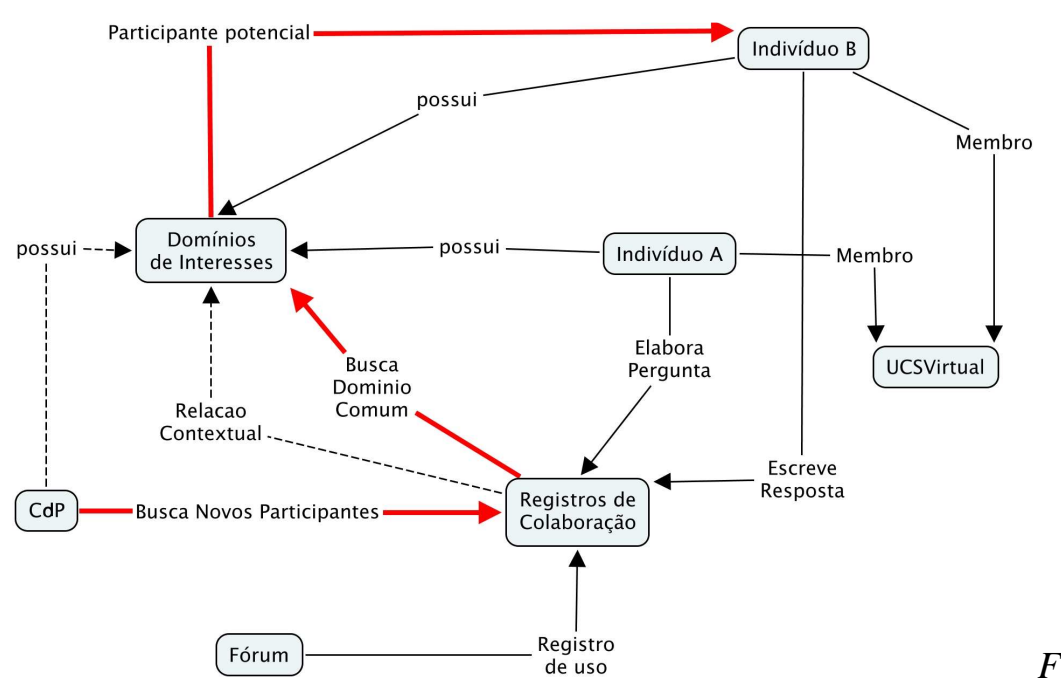

igura 3: Exemplo de busca de possíveis participantes para a CdP

A busca realizada pela CdP para encontrar possíveis novos participantes pode ser realizada pelo caminho inverso, ou seja, indivíduos buscando comunidades com os mesmo interesses. As buscas também podem ser direcionadas aos registros das ferramentas, indivíduos buscando documentos, discussões, respondendo questões ou solucionando problemas.

\subsection{Utilizando o Protótipo}

Um protótipo para experimentos foi implementado usando o Sistema de Gestão de Conteúdo Plone ${ }^{1}$. O cenário utilizado define duas CdPs com 10 participantes em torno dos Domínios de Interesse: "Ensino à Distância" e "Zoologia". A Figura 4 ilustra a interface geral da CdP, especificamente para o domínio de "Ensino à Distância", que será chamada de CdP-EAD.

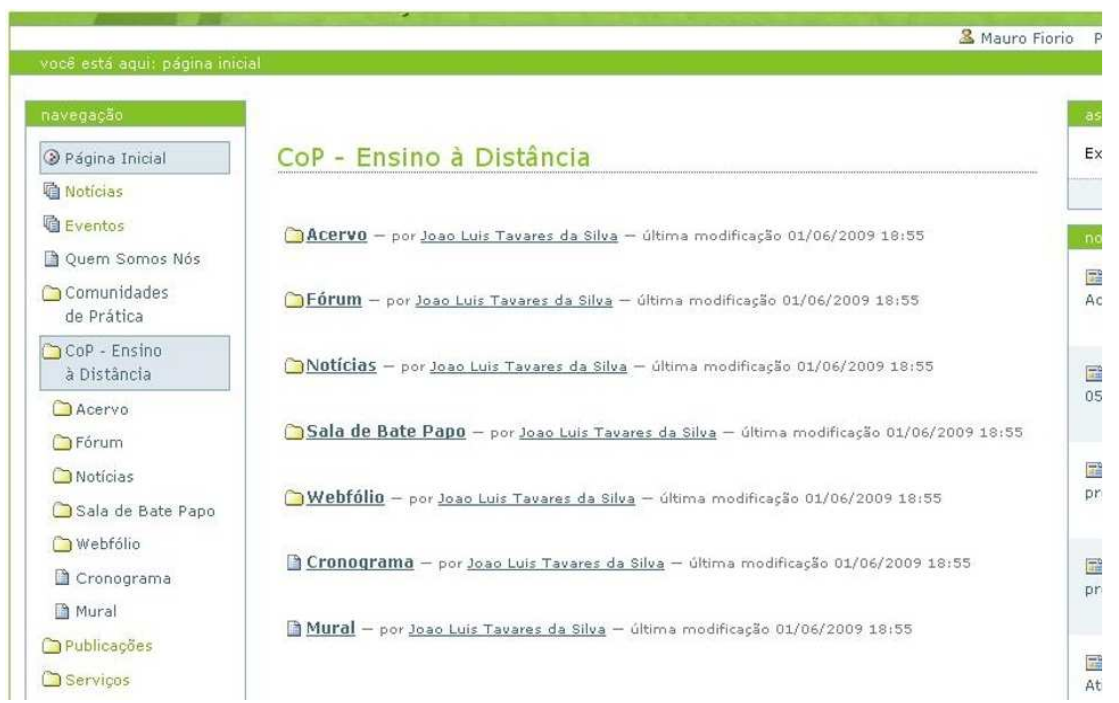

Figura 4: Exemplo da interface para o protótipo de CdP.

O processo de registro da CdP permite que o usuário escreva as características da comunidade e convide algumas pessoas para participar. $\mathrm{O}$ conhecimento veiculado

${ }^{1} \mathrm{Http} / / /$ plone.org

V. $9 \mathrm{~N}^{\circ} 1$, julho, 2011 
pelos usuários fica registrado nas classes Domínios de Interesse e Registros de Colaboração. A Figura 5 ilustra uma parte do registro destas informações no banco de dados da $\mathrm{CdP}$, relacionando os participantes e a relação entre o perfil da $\mathrm{CdP}$ e os domínios de interesse de duas CdP distintas.

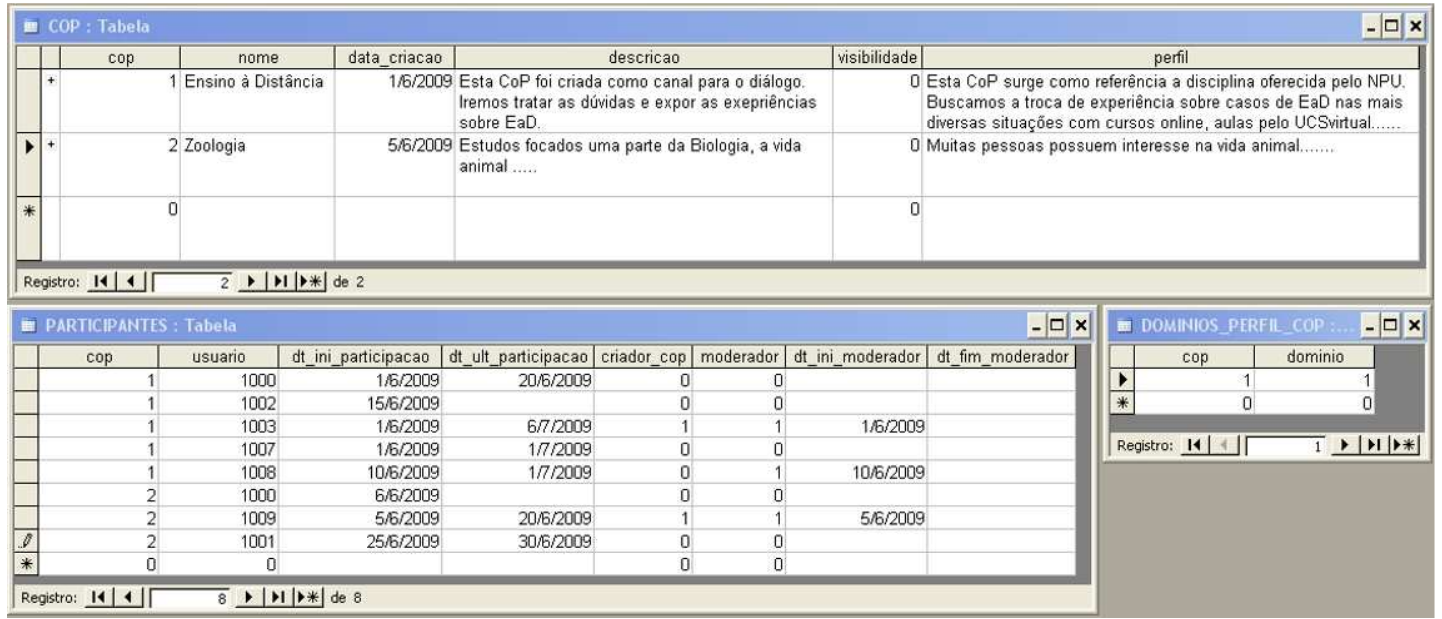

Figura 5: Exemplo das tabelas de dados para o cadastro da CdP, Participantes e Domínios de Interesse relacionados.

Foi criado um fórum sobre "Mestrado a Distância" (Figura 6) apresentando apenas um código identificador da ferramenta, o usuário e sua opinião.

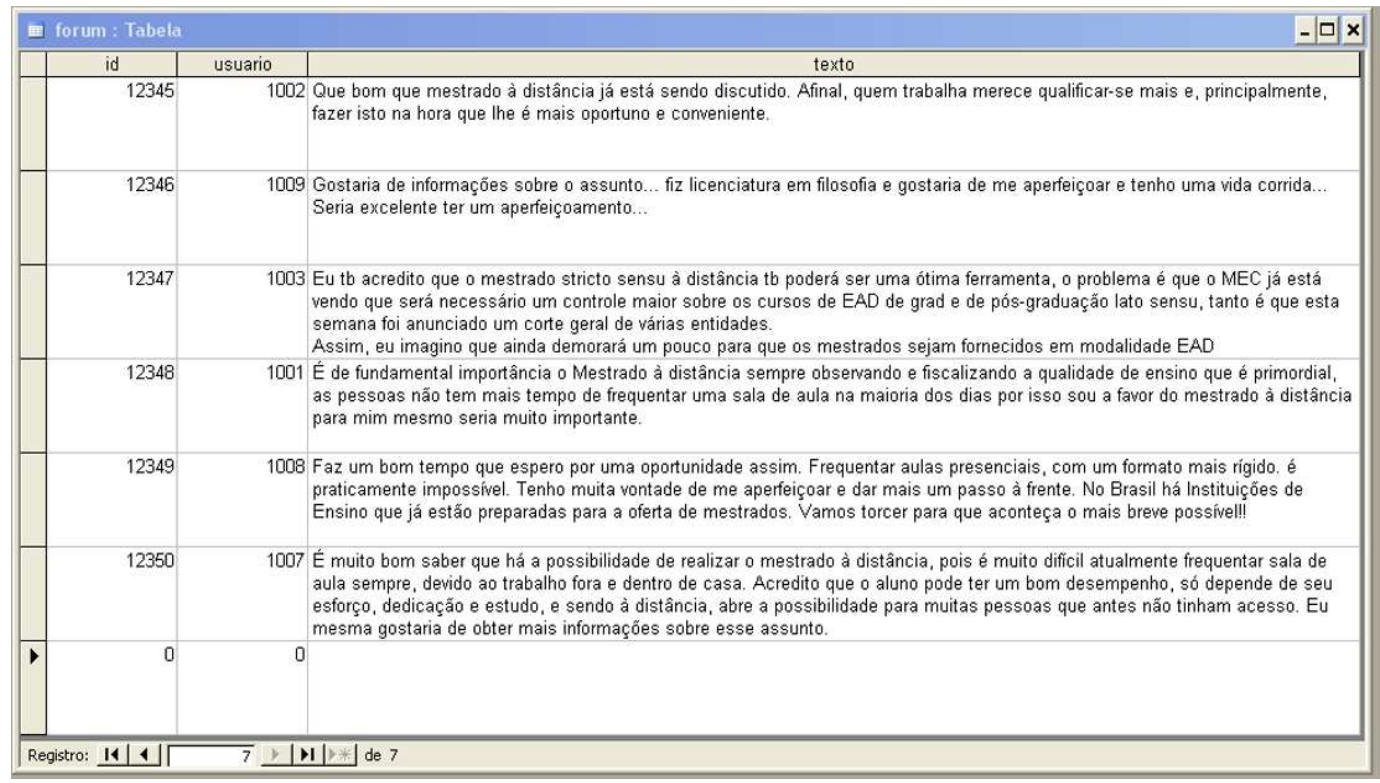

Figura 6: Abstração do armazenamento do Fórum nas tabelas de dados do protótipo.

A utilização do Fórum gera uma série de registros nas tabelas do UCSVirtual armazenando os diálogos e os usuários envolvidos nestas conversas. Estes, são registrados no Registro de Colaboração vinculando o identificador da ferramenta, a CdP e os domínios de interesse correspondentes. Neste artigo, não serão abordados todos os itens associados aos domínios de interesse, porém vale lembrar que nos casos de inserção de novos domínios uma rotina verifica as informações existentes e constrói as relações entre as informações e o novo domínio. O Registro de Colaboração serve 
essencialmente para manter a associação dos eventos das ferramentas colaborativas com os domínios de interesse.

Tomando apenas o domínio de interesse "Ensino à Distância" como cenário de análise, podemos perceber neste conjunto de dados que:

- dois indivíduos (códigos 1003 e 1008), declaram no seu perfil o interesse pelo mesmo domínio e participam de uma CdP (CdP-EAD) com o mesmo interesse;

- os dois indivíduos 1001 e 1004 possuem interesse no domínio da CdP mas não participam, tornando-se possíveis participantes em potencial;

- os participantes 1002 e 1007 não possuem o domínio em seu perfil mas participaram das ferramentas colaborativas;

- o usuário 1001 não é participante da CdP-EAD mas possui interesse no domínio através de sua participação e perfil, podendo ser um potencial participante;

- o usuário 1008 tem o domínio relacionado com seu perfil e também participa da CdP-EAD, mas não possui registro de colaboração. Este usuário pode ser estimulado por meio de um convite para colaborar;

- os registros de colaboração estão associados a CdP correta, uma vez que a comunidade e os registros tratam do mesmo domínio.

Oferecer suporte ao UCSVirtual é, entre outras atividades, permitir que análises, como a anterior, sejam realizadas pelos moderadores das CdPs. Quando um moderador possui tais dados em mãos, ele pode tomar decisões que promovem o conhecimento e o trabalho colaborativo dos usuários do UCSVirtual.

\section{CONCLUSÕES}

Apresentamos neste trabalho um estudo das necessidades e a especificação de requisitos que envolvem a implantação de Comunidades de Prática no AVA do UCSVirtual.

Neste trabalho, elaboramos a hipótese de que uma abordagem baseada em CdP pode servir de suporte e estímulo ao trabalho colaborativo no compartilhamento de conhecimento em comunidades virtuais. Esta hipótese sugere que as ferramentas colaborativas, agregadoras de relações interpessoais e de conteúdo, podem expandir a dimensão pedagógica e colaborativa dos envolvidos no ambiente.

O framework apresentado oferece um conjunto de estruturas e funcionalidades que garantem que esta $\mathrm{CdP}$, depois de devidamente cadastrada, possa se autogerir (através dos moderadores) e compartilhar conhecimentos com novos participantes, indiferentemente da participação em uma ou outra comunidade. Além das funcionalidades de gestão, o framework também define a identificação da frequência das atividades da $\mathrm{CdP}$, observando se as atividades propostas são realizadas por participantes ou usuários do AVA. É possível realizar verificações como: quais são as CdP que tratam de um assunto específico ou quais são os usuários que gostam deste assunto. Através das $\mathrm{CdP}$ os conhecimentos vão sendo compartilhados entre as pessoas mesmo que estas tenham interesse no assunto em momentos diferentes.

Estamos constituindo desta forma, os passos iniciais para uso de uma abordagem de Web Semântica em Ambientes de Aprendizagem, oferecendo recursos inteligentes de troca de conhecimento e gestão do aprendizado coletivo. 


\section{REFERÊNCIAS}

Albagli, S. (2007). Tecnologias da Informação, Inovação e Desenvolvimento. VII Cinform. Encontro Nacional de Ciência da Informação.

Casa, M. E., \& Ribeiro, A. M. (2005). Ambientes Inteligentes de Aprendizagem. In E. M. Valentini, Carla Beatris; Soares, Aprendizagem em Ambientes Virtuais: compartilhando idéias e construindo cenários (1 ed., pp. 227-239). Caxias do Sul: EDUCS.

Dillenbourg, P. (2000). Virtual Learning Environments. In EUN Conference 2000: Learning in the new millennium: building new education strategies for schools (pp. 1-30).

Gongla, P., \& Rizzuto, C. R. (2001). Evolving Communities of Practice: IBM Global Services experience. IBM Systems Journal, 40(4), 842.

IBM. (2008). Building a Transformative Network: IBM Virtual Learning Community Case Study.

Kanter, R. M. (2001). Evolve!: Succeeding in The Digital Culture of Tomorrow. In Gestão do Conhecimento: on knowledge management. Harvard Business School.

Pereira, R., \& Neves, P. (2006). As Comunidades de Prática como Instrumento de desburocratização da Administração local e de sustentação dos objetivos dos projectos cidades e regiões digitais.

Terra, J. C. (2005). Comunidades de Prática: conceitos, resultados e métodos de gestão. Terra Forum.

WORDBANK. (2008). Communities of practice for development in the Middle East and North Africa.

Wenger, E. (1998). Communities of Practice: learning, meaning and identity. Cambridge: Cambridge University Press, 1998.

Wenger, E. (2008). Communities of practice: a brief introduction. Disponível em: http://www.ewenger.com/theory/communities_of_practice_intro.htm. Acesso em maio de 2011.

Wenger, E., Mcdermott, R., \& Snyder, W. (2002). Cultivating communities of practice: A guide to managing knowledge.. Boston: Business School Press.

Wenger, E., White, N., \& Smith, J. D. (2005). Technology for Communities. Disponível em: http://pt.scribd.com/doc/2531741/Technology-for-communities-Wenger-CEFRIO-BookChapter-v-5-2. Acesso em abril de 2011.

XEROX. (2005). Award Recipient Rank Xerox (Hong Kong) Ltd. 